\title{
The Reliability of Ultrasound Measurements of Carotid Stenosis Compared to MRA and DSA
}

\author{
Colin Honish, Venkatraman Sadanand, Derek Fladeland, Vance Chow, \\ Fahrad Pirouzmand
}

\begin{abstract}
Objective: Carotid ultrasound (US) is a screening test for patients with transient ischemic attacks (TIAs) or stroke who then undergo Digital Subtraction Angiogram (DSA) or Magnetic Resonance Angiography (MRA). Gold standard DSA is invasive with inherent risks and costs. MRA is an evolving technology. This study compares reliability of MRA and US modes with DSA in determining degree of internal carotid artery stenosis. Methods: A five year retrospective analysis of 140 carotid arteries from patients who had carotid US and DSA, and possibly Magnetic Resonance Angiography was undertaken. Recorded US parameters were peak systolic velocity (PSV), end diastolic velocity (EDV), and ICA/CCA peak systolic velocity ratio. The MRA and DSA parameters used NASCET technique for measuring stenosis. Statistical analysis included ROC curves and Kappa computation. Results: US grading of carotid stenosis can be made more reliable by choosing appropriate parameters. The best combination of sensitivity and specificity for stenosis $>70 \%$ in our hospital was seen at PSV $>173 \mathrm{~cm} / \mathrm{s}$ (sensitivity 0.87 , specificity 0.8, Positive Predictive Value (PPV) 0.70 , Negative Predictive Value (NPV) 0.93, kappa 0.64 and weighted kappa 0.71). MRA kappa was 0.78 , (sensitivity 0.75 , specificity 1.0 , PPV 1.0, NPV 0.85). Conclusions: US parameters should be validated in each centre. At best, US can only approximate the accuracy of DSA, probably due to inherent limitations of this modality. Magnetic Resonance Angiography has a perfect specificity and PPV but this technique needs to be standardized. Simultaneous use of MRA and US for screening increases sensitivity to over 0.9 without compromising specificity in $>70 \%$ stenosis.
\end{abstract}

RÉSUMÉ: Comparaison de la fiabilité des mesures de la sténose carotidienne par échographie, ARM et AN. Objectif: L'échographie carotidienne (ÉC) est une épreuve de dépistage chez les patients qui ont subi une ischémie cérébrale transitoire (ICT) ou un accident vasculaire cérébral (AVC) et chez qui on pratique ensuite un angiogramme numérisé (AN) ou un angiogramme par résonance magnétique (ARM). L'étalon or est l'AN qui est un examen effractif avec les risques et les coûts qui y sont associés. L'ARM est une technique en développement. Cette étude compare la fiabilité de l'ARM et de l'ÉC par rapport à l'AN pour déterminer le degré de sténose de la carotide interne (CI). Méthodes: Il s'agit d'une étude rétrospective de 140 artères carotides de patients qui ont subi une ÉC et un AN et dont certains ont subi une ARM. Les paramètres enregistrés à l'ÉC étaient la vélocité systolique maximale (VSM), la vélocité en fin de diastole (VFD) et le rapport de la vélocité systolique maximale dans la carotide interne sur celle de la carotide commune (RV). Les critères NASCET ont été utilisés pour mesurer la sténose au moyen des paramètres obtenus par ARM et AN. L'analyse statistique comportait la détermination de la courbe ROC et du coefficient Kappa. Résultats: La fiabilité de la détermination du degré de sténose carotidienne par ÉC peut être améliorée par le choix de paramètres appropriés. La meilleure combinaison de sensibilité et de spécificité pour une sténose $>70 \%$ dans notre hôpital a été observée à une VSM $>173 \mathrm{~cm} / \mathrm{s}$ (sensibilité 0,87 ; spécificité 0,8 ; valeur prédictive positive (VPP) 0,70 valeur prédictive négative (VPN) 0,93, Kappa 0,64 et Kappa pondéré 0,71). La valeur Kappa pour l'ARM était de 0,78 (sensibilité 0,75; spécificité 1,0 ; VPP 1,0 et VPN 0,85). Conclusions: Les paramètres de l'ÉC devraient être validés dans chaque centre. Au mieux, l'ÉC ne peut qu'approcher la précision de l'AN, probablement à cause des limites inhérentes à cette technique. L'ARM a une spécificité et une VPP parfaites, mais cette technique doit être standardisée. L'utilisation simultanée de l'ARM et de l'ÉC pour le dépistage augmente la sensibilité à plus de 0,9 sans compromettre la spécificité pour les sténoses $>70 \%$.

Carotid Doppler ultrasounds (US) are routinely performed as noninvasive screening tests on patients who present with TIA's or strokes. ${ }^{1}$ Many of these patients then undergo Digital Subtraction Angiogram (DSA) or Magnetic Resonance Angiography (MRA). Digital Subtraction Angiogram is invasive with inherent risks and financial costs. ${ }^{2-4}$ Magnetic Resonance Angiography is an evolving technology ${ }^{5}$ with unsubstanitiated reliability ${ }^{6}$ and low sensitivity resulting in the possibility of unacceptable false negatives. ${ }^{7,8} \mathrm{We}$ performed a retrospective
From the Department of Neurosurgery, University of Saskatchewan (CH, VS, DF, VC), Royal University Hospital, Saskatoon, SK, Canada; Department of Neurosurgery, University of Toronto (FP), Sunnybrook and Women's Health Sciences Center, Toronto, ON, Canada.

Received OCtober 18, 2004. AcCePted in final form August 5, 2005. Reprint requests to: Farhad Pirouzmand, University of Toronto, Sunnybrook and Women's Health Sciences Center, 2075 Bayview Avenue, Toronto, Ontario, Canada M4N 3M5 
analysis comparing the reliability of MRA and US with the "gold standard" of DSA in determining the degree of stenosis.

Computed Tomography Angiography (CTA) has recently become an excellent option for screening and diagnosing internal carotid artery (ICA) stenosis. ${ }^{9}$ Computed Tomography Angiography will likely become the main screening/ diagnostic test for many vascular diseases, and will likely replace angiogram in the future. In our centre, however, angiography is still the gold standard. There are several limitations to CTA because not all centres have multidetector computed tomagram scanners, and technologist training required for adequate use of CTA may be in short supply. This will likely change with time, but for now US is still a main screening test in our centre.

US measures stenosis indirectly through velocity of blood flow $^{10}$ using the Bernoulli principle. ${ }^{11}$ Digital Subtraction Angiography (DSA) and MRA both measure stenosis directly using respective images. When interpreting US velocities, standard values and cutoff points must be available to reference degree of stenosis. These values will change from hospital to hospital, depending on US machines and technicians. ${ }^{12}$ It is important for each centre to be aware of operator dependent factors that may contribute to the inaccuracies of US in measuring stenosis. ${ }^{13}$ US can be made more reliable by choosing center specific, statistically validated, cutoff parameters for grading carotid stenosis.

The objectives of this study are as follows:

1. To find the optimal US cutoff parameters for our institution, mainly to stress the importance of validation of these values.

2. To stress the importance of use of weighted kappa in optimizing cutoff parameter values.

3. To compare our US and MR results with the gold standard angiogram test.

\section{Methods}

We conducted a five-year (1998-2002) retrospective analysis of symptomatic patients who had US followed by carotid Digital Subtraction Angiography (DSA) in single hospital (the Royal University Hospital, Saskatoon, Saskatchewan, Canada). Some of the patients had MRA as well. We collected data on the degree of stenosis using North American Symptomatic Carotid Endarterectomy (NASCET) ${ }^{14,15}$ criteria on 140 carotid arteries. The measured US parameters were Peak Systolic Velocity (PSV), End Diastolic Velocity (EDV), and ICA:CCA PSV velocity ratio (VR). Together, these are commonly used for predicting US stenosis. ${ }^{16}$ MRA degree of stenosis was evaluated by an independent radiologist (blinded to the US result) using North American Symptomatic Carotid Endarterectomy Trial (NASCET) criteria and clinical judgment. ${ }^{17}$

Ultrasound films were reviewed by two authors ( $\mathrm{CH}$ and FP), and the maximal PSV and EDV from the ICA views of the stenosis were recorded. Doppler waveforms of ICA blood flow was determined by the US machines used, which also directly converted the waveforms to velocities. From these US films VR was calculated and degree of stenosis was determined using our institution parameter values. Plaque characteristics ${ }^{18,19}$ such as morphology (ulceration, irregular or smooth plaques) and location of maximal plaque formation were also determined from US films in our study, but there will be no attempt to analyze this data in this study.

All MRA images were acquired on a 1.5T Siemens Symphony system (Siemens Medical Systems, Iselin, NJ). Images were acquired just after hand injection of $0.1 \mathrm{mmol} / \mathrm{kg}$ of gadoteridol (ProHance, Bracco Diagnostics, Princeton NJ) at a rate of about $2 \mathrm{cc} / \mathrm{sec}$ followed by a saline flush. The sequence used was a breath-hold three dimensional fast low angle shot obtained in the coronal plane. After MRA images were obtained, the degree of stenosis was determined by a single radiologist using their clinical judgement as well as measurements of the diameter of maximal stenosis in relation to the distal normal ICA diameter as per NASCET criteria. The equation used to determine percent stenosis is expressed below.

Digital Subtraction Angiography offers multiple views and angles of the carotid vessels. For our study, the view with the narrowest diameter of stenosis of the vessel was used to determine percent stenosis. DSA measures stenosis in terms of pixels, not centimeters, and in our study we determined the percent stenosis by using the equation: ${ }^{17}$

1 - (maximal stenosis diameter in pixels) (distal normal segment diameter in pixels)

x $100 \%$

Digital Subtraction Angiography stenosis was determined from radiologist reports as well as reviewing each angiogram to confirm their measurements. For some of the vessels where there was controversy between our measurement and the radiologist report, or where some were difficult to measure, we used digitized pictures of the maximal stenosis to determine percentage. We then used a computer graphics program (Canvas 7, Deneba Systems Inc. Miami, Florida), following NASCET criteria, to determine accurate measurements of stenosis. These DSA diameters were measured in pixels after taking a close up digital photograph of the narrowest view of the vessel, and measuring the stenotic diameter using computer measurements of diameter.

\section{The inclusion criteria for our study were:}

1. Carotid angiogram done for symptomatic carotid disease including TIA's strokes, amaurosis fugax, reversible ischemic neurologic deficit, arterio-venous malformations, and syncope, as seen below (Table 1).

\section{Table 1: Reason For Patient Referral}

\begin{tabular}{|c|c|c|}
\hline Reason for Referral & Frequency & Percent \\
\hline TIA & 39 & 47.6 \\
\hline Stroke & 34 & 41.5 \\
\hline Amaurosis Fugax (TIA) & 4 & 4.9 \\
\hline RIND & 2 & 2.4 \\
\hline AVM & 1 & 1.2 \\
\hline Ischemic Ocular Syndrome & 1 & 1.2 \\
\hline Syncope & 1 & 1.2 \\
\hline TOTALS & 82 & 100 \\
\hline \multicolumn{2}{|l}{} \\
\hline
\end{tabular}


Table 2: Frequency and Location of Carotid Stenosis Lesions Based on the US result

\begin{tabular}{|l|l|}
\hline LOCATION & FREQUENCY \\
\hline Proximal ICA & 106 \\
\hline Middle ICA & 23 \\
\hline Distal ICA & 2 \\
\hline Carotid bulb & 6 \\
\hline Unable to Visualize & 8 \\
\hline No Stenosis & 17 \\
\hline CCA before bulb & 2 \\
\hline TOTAL & 164 \\
\hline
\end{tabular}

2. Carotid Doppler ultrasound preceding angiogram.

3. Magnetic Resonance Angiography of carotids if available. The MRA was always performed after US suspected the stenosis.

Eighty-two patients were identified that met our criteria. Although all patients were symptomatic, there were carotids studied which had no symptoms attributed to them. These were contralateral to the symptomatic side. All stenotic lesions were near the carotid bifurcation (Table 2). Proximal ICA stenosis was $<2 \mathrm{~cm}$ from the bifurcation, middle ICA stenosis was $2-5 \mathrm{~cm}$, and distal ICA was $>5 \mathrm{~cm}$ from the bifurcation. There have been other studies focusing on carotid endarterectomy for treatment of asymptomatic carotid stenosis, ${ }^{20}$ as well as symptomatic carotid stenosis, ${ }^{14}$ and our methods are somewhat similar to these in determining how US correlates to DSA.

Of the 164 arteries, 156 were visualized adequately enough in DSA to compute stenosis using NASCET criteria. Of these 156, 16 were removed from analysis because carotid US showed complete or near complete occlusions. These occlusions were excluded from the study for the purpose of mathematical calculations as the PSV and/or EDV will be $0 \mathrm{~cm} / \mathrm{s}$ (no flow) or near $0 \mathrm{~cm} / \mathrm{s}$. Just preceding complete occlusion, the velocities would be very high. This creates a discontinuity and will invalidate the correlation calculations.

The number of carotid arteries studied by MRA in our study was 23 , of which 19 were adequately visualized to determine a degree of stenosis.

\section{Statistical Analysis}

We used the program SPSS-10 for data analysis. The optimal cutoff points for sensitivities and specificities of various US parameters (PSV, EDV, VR) were determined from receiver operating characteristic (ROC) curves, and these were compared to the current parameters used at our institution (Royal University Hospital) (see Table 3).
Table 3: Current Parameters for Carotid Ultrasound degree of Stenosis used at Royal University Hospital.

\begin{tabular}{|c|c|c|c|}
\hline Degree of Stenosis & PSV $(\mathrm{cm} / \mathrm{s})$ & EDV $(\mathrm{cm} / \mathrm{s})$ & ICA:CCA PSV ratio \\
\hline$<40 \%$ & $<125$ & - & - \\
\hline $40-70 \%$ & $125-230$ & - & $<3.2$ \\
\hline $70-90 \%$ & $>230$ & $>70$ & $>3.2$ \\
\hline$>90 \%$ & $>230$ & $>125$ & - \\
\hline
\end{tabular}

We calculated Kappa and Weighted Kappa values as measures of correlation between US or MRA, versus DSA. Kappa is a chance-corrected measure of agreement, which considers both proportion of observed agreement and proportion of agreement by chance. Kappa agreement has been described to be excellent if $>0.8$ ( $80 \%$ agreement), substantial if $>0.6$ $(60 \%)$, moderate if from $0.4-0.6(40-60 \%)$, and poor if $<0.4$ $(<40 \%){ }^{21}$

Asymmetrically Weighted Kappa, described by Portney ${ }^{21}$ is used to differentiate among disagreements. Not all disagreements are of equal weight and some misclassifications would be more serious than others. In our study, the risk is more serious if a patient had undiagnosed carotid stenosis (false negative US) than the risk of unnecessary angiogram (if they had a false positive US result). Thus, the highest weight of 4 was assigned to false negative US results, based on our subjective assessment of the relative importance involved in false US results. ${ }^{21,22}$ The false positive errors received a weight of (1). True positive and true negative values were given a weight of (0). These different weight assignments allows us to assign importance to the direction of disagreement.

The MRA calculations were based on agreement between the blinded radiologist's interpretation of degree of stenosis and angiogram NASCET degree of stenosis. Sensitivity, specificity, Positive Predictive Value (PPV), Negative Predictive Value (NPV), kappa, and weighted kappa were also obtained when comparing MRA with DSA.

Table 4: Angiogram Prevalence of Degree of Stenosis

\begin{tabular}{|c|c|c|}
\hline CATEGORY & PREVALENCE & PERCENT (\%) \\
\hline$<30 \%$ Stenosis & $45 / 140$ & 15 \\
\hline $30-49 \%$ Stenosis & $21 / 140$ & 18.6 \\
\hline $50-69 \%$ Stenosis & $26 / 140$ & 22.9 \\
\hline $70-89 \%$ Stenosis & $32 / 140$ & 3.6 \\
\hline $90 \%$ Stenosis & $5 / 140$ & 7.9 \\
\hline$>95 \%$ Stenosis & $11 / 140$ & \\
\hline
\end{tabular}




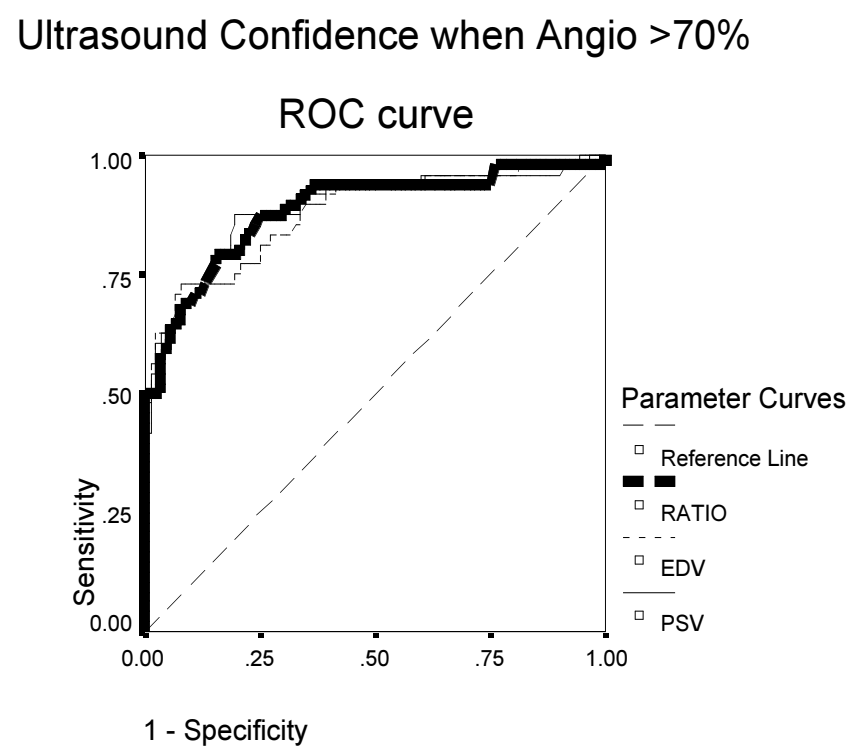

Figure: ROC Curve to Determine Sensitivity / Specificity of US Parameters PSV, EDV, VR for Stenosis $>70 \%$.

\section{RESULTS}

One hundred and sixty four carotid arteries from 82 patients were studied with both US and DSA, with some having MRA. Of the 164 arteries, 156 were adequately visualized to give a NASCET degree of stenosis. There were 16 occlusions or near occlusions of the $156(10.3 \%)$ which were excluded for mathematical reasons. The remaining 140 are classified in Table 4.

On angiogram, $34.4 \%$ of patients had high grade stenosis $(\geq 70 \%)$, and $18.6 \%$ had moderate grade stenosis (50-69\%).

\section{Ultrasound Results}

Using ROC curves (Figure, Table 5) the best combination of sensitivity and specificity for stenosis $>70 \%$ was seen at PSV of $173 \mathrm{~cm} / \mathrm{s}$ yielding a sensitivity of $87.5 \%$, specificity of 80.4\%, PPV 0.70, NPV 0.93, Kappa 0.641, and Weighted Kappa 0.718 (Table 6).

With the current parameters used at our institution for stenosis $>70 \%$, the PSV cutoff of $230 \mathrm{~cm} / \mathrm{s}$ yielded the following: sensitivity $68.8 \%$, specificity $90.2 \%$, PPV 0.78 , NPV 0.85, Kappa 0.608, and Weighted Kappa 0.574 (Table 6). Thus this type of analysis showed that our current parameters for PSV were suboptimal. The current parameter values of EDV $>70 \mathrm{~cm} / \mathrm{s}$ and VR > 3.2 were shown to have higher values of agreement than our data values of EDV $>54 \mathrm{~cm} / \mathrm{s}$ and $\mathrm{VR}>1.9$, as measured by kappa and weighted kappa. This suggests that the current parameters for EDV and VR are optimal.

In our study, we correlated agreement between US and DSA for high grade stenosis $>70 \%$ (Table 6), and stenosis $>40 \%$ (Table 7). The ROC curves for angiogram stenosis $>70 \%$ were determined using US parameters PSV, EDV and VR, (as seen in Figure, Table 5). Similar figures and tables for stenosis $>40 \%$ were obtained but are not included in this paper for the sake of brevity.
Table 5: Sensitivity / Specificity combinations for US PSV parameters when Angio $\mathbf{7 0 \%}$.

\begin{tabular}{lll}
\hline PSV Value & Sensitivity & 1-Specificity \\
& & \\
165.5000 & .875 & .217 \\
168.0000 & .875 & .207 \\
$\mathbf{1 7 3 . 0 0 0 0}$ & $\mathbf{. 8 7 5}$ & $\mathbf{. 1 9 6}$ \\
176.5000 & .854 & .196 \\
180.0000 & .833 & .185 \\
185.5000 & .813 & .185 \\
191.5000 & .792 & .185 \\
198.5000 & .792 & .163 \\
204.8500 & .771 & .163 \\
208.3500 & .771 & .152 \\
211.5000 & .750 & .141 \\
214.3000 & .750 & .130 \\
215.3000 & .729 & .130 \\
218.5000 & .729 & .120 \\
221.6000 & .708 & .120 \\
224.1000 & .708 & .109 \\
226.5000 & .708 & .098 \\
$\mathbf{2 3 2 . 0 0 0 0}$ & $\mathbf{. 8 8 8}$ & .098 \\
241.0000 & .688 & .087 \\
249.0000 & .667 & \\
\hline
\end{tabular}

\section{MRA Results}

Of the 23 carotid vessels imaged by MRA, 19 were adequately visualized. The 19 MRA vessels had a kappa correlation with DSA of 0.78 , (sensitivity $75 \%$, specificity $100 \%$, PPV $100 \%$, NPV 85\%).

Table 6: US Parameter Value Comparison Between Our Data Values and Current Hospital Values for Stenosis $\mathbf{> 7 0 \%}$

\begin{tabular}{|c|c|c|c|c|c|c|}
\hline & \multicolumn{2}{|c|}{ PSV } & \multicolumn{2}{|c|}{ EDV } & \multicolumn{2}{|c|}{ VR } \\
\hline & $\begin{array}{l}\text { Our Data } \\
>142.45 \\
(\mathrm{~cm} / \mathrm{s})\end{array}$ & $\begin{array}{l}\text { Current } \\
\text { Cut-off } \\
>125(\mathrm{~cm} / \mathrm{s})\end{array}$ & \begin{tabular}{|l} 
Our Data \\
$>43.75$ \\
$(\mathrm{~cm} / \mathrm{s})$
\end{tabular} & $\begin{array}{l}\text { Current } \\
\text { Cut-off } \\
\text { NONE }\end{array}$ & $\begin{array}{l}\text { Our } \\
\text { Data } \\
>1.55\end{array}$ & $\begin{array}{l}\text { Current } \\
\text { Cut-off } \\
>1.6\end{array}$ \\
\hline SENSITIVITY (\%) & 77.4 & 83 & 76.2 & - & 79.8 & 77 \\
\hline SPECIFICITY (\%) & 84 & 68 & 80.4 & - & 80.4 & 82 \\
\hline PPV (\%) & 88 & 80 & 85 & - & 86 & 87 \\
\hline NPV (\%) & 71 & 73 & 69 & - & 73 & 70 \\
\hline KAPPA & 0.595 & 0.518 & 0.551 & - & 0.591 & 0.580 \\
\hline
\end{tabular}

PSV $=$ Peak Systolic Velocity, PPV = Positive Predictive Value, EDV = End Diastolic Velocity, NPV $=$ Negative Predictive Value, VR $=$ PSV ICA: PSV CCA - Velocity Ratio, KAPPA = Kappa correlation coefficient, Weighted Kappa = Value of 4 was fiven to false negative results using Asymmetrical weighting as described by Portney. 


\section{Sequential or Simultaneous Testing}

In our study, MRA was always performed after US suspected an area of stenosis, and this form of testing is truly sequential in nature. Using US (PSV $>173 \mathrm{~cm} / \mathrm{s}$ ) and MRA together as sequential two stage screening for stenosis $>70 \%$, the net sensitivity was $65.6 \%$, while net specificity was $100 \%$. For US (PSV $>230 \mathrm{~cm} / \mathrm{s}$ ) and MRA together as sequential two stage screening for stenosis $>70 \%$, the net sensitivity was $51 \%$, while net specificity was $100 \%$. Conversely, using US (PSV > $173 \mathrm{~cm} / \mathrm{s}$ ) and MRA together as simultaneous screening for stenosis $>70 \%$, the sensitivity theoretically could be raised to 96\% (assuming US and MRA result are independent) while specificity is maintained at $80.4 \%$.

\section{Discussion}

Carotid Doppler ultrasound is a routine screening test for patients presenting with TIAs or strokes. Due to the poor image quality of ultrasounds, the degree of stenosis is indirectly measured using blood flow velocities just distal or proximal to the plaque. This measurement is then used to classify the plaque's effect as mild, moderate or severe stenosis. Thus there are two principles used in this process: first, velocities are inferred from ultrasound frequency changes using the Doppler effect, and second, stenosis is inferred from velocities using the Bernoulli principle. ${ }^{11}$ Blood flow through the carotid proximal and distal to the stenosis is equal. As blood moves from a wider proximal carotid into a stenotic region the same mass flow rate must be maintained in accordance with the Equation of Continuity. ${ }^{11}$ The same volume must therefore move through a

Table 7: US Parameter Value Comparison Between Our Data Values and Current Hospital Values for Stenosis $>40 \%$

\begin{tabular}{|c|c|c|c|c|c|c|}
\hline & \multicolumn{2}{|c|}{ PSV } & \multicolumn{2}{|c|}{ EDV } & \multicolumn{2}{|c|}{ VR } \\
\hline & $\begin{array}{l}\text { Our Data } \\
>142.45 \\
(\mathrm{~cm} / \mathrm{s}) \\
\end{array}$ & \begin{tabular}{|l} 
Current \\
Cut-off \\
$>125(\mathrm{~cm} / \mathrm{s})$ \\
\end{tabular} & \begin{tabular}{|l|} 
Our Data \\
$>43.75$ \\
$(\mathrm{~cm} / \mathrm{s})$ \\
\end{tabular} & $\begin{array}{l}\text { Current } \\
\text { Cut-off } \\
\text { NONE } \\
\end{array}$ & \begin{tabular}{|l|} 
Our \\
Data \\
$>1.55$ \\
\end{tabular} & $\begin{array}{l}\text { Current } \\
\text { Cut-off } \\
>1.6 \\
\end{array}$ \\
\hline SENSITIVITY (\%) & 77.4 & 83 & 76.2 & - & 79.8 & 77 \\
\hline SPECIFICITY (\%) & 84 & 68 & 80.4 & - & 80.4 & 82 \\
\hline PPV (\%) & 88 & 80 & 85 & - & 86 & 87 \\
\hline NPV (\%) & 71 & 73 & 69 & - & 73 & 70 \\
\hline KAPPA & 0.595 & 0.518 & 0.551 & - & 0.591 & 0.580 \\
\hline
\end{tabular}

PSV $=$ Peak Systolic Velocity, PPV = Positive Predictive Value, EDV = End Diastolic Velocity, NPV = Negative Predictive Value, VR = PSV ICA: PSV CCA - Velocity Ratio, KAPPA = Kappa correlation coefficient smaller cross section. Thus the flow velocity in the constriction must be greater than in the proximal carotid.

The indirect inferential grading of stenosis necessarily involves the subjective judgment of technician and radiologist. This could possibly result in inaccuracies. Furthermore the cutoff parameters for ultrasound grading of stenosis at a center may require validation based on DSA and MRA data. The current gold standard for measuring carotid stenosis is the DSA. However, this technique is invasive and carries a small risk of stroke, dissection, pseudoaneurysm formation and death. It is also more expensive than a Doppler ultrasound. The MRA is relatively new and does produce good quality images depending on the scanning sequence. Various scanning techniques are evolving at present. Once a professionally accepted standard for carotid MRAs is established, it may prove to be of acceptable accuracy.

Using simple retrospective data, it is easy to validate the current cutoff parameters for ultrasound grading of carotid stenosis. In the present study, it is seen that the existing parameters are suboptimal and a lower PSV, for example, would yield more accurate classification.

We excluded complete or near complete occlusions from our mathematical calculations because Doppler velocities become discontinuous when approaching $100 \%$ stenosis. For example, while $70 \%$ stenosis may have a PSV of $>230 \mathrm{~cm} / \mathrm{s}$, and a $95 \%$ narrowing may have a PSV of around $390 \mathrm{~cm} / \mathrm{s}$, a complete occlusion will have a PSV of $0 \mathrm{~cm} / \mathrm{s}$, and a near complete occlusion may have an EDV dropping to $0 \mathrm{~cm} / \mathrm{s}$. In these situations the blood velocities do not carry useful information and the physician should recognize the possibility of complete or near complete occlusion. Individual clinical judgment provides the best interpretation.

The above example shows how US is an indirect measure of degree of stenosis. Flow velocities do not equal percent stenosis, but they can be indirectly correlated to percent stenosis using the Bernoulli principle. This is an inherent limitation of Doppler ultrasound, because while MRA and DSA can directly measure degree of stenosis using NASCET criteria, US can only correlate flow velocities to stenosis. Thus validation of US parameters is essential to increase the accuracy of this modality.

The investigations in our study were all done for the purpose of diagnosis, and all ultrasounds performed were for diagnosis and not screening. This is an important point because true screening of carotids would involve asymptomatic patients, and since our patients all were symptomatic, our population is biased. The asymptomatic carotid atherosclerosis study (ACAS) trial specifically looked at asymptomatic patients, and thus their investigations were true screening tests, while our population focused on diagnostic tests.

As mentioned above, our methods are somewhat similar to ACAS and NASCET studies in determining how US correlates to DSA. However, our study is quite different from these in that our study is a small retrospective analysis of data at our institution, used to try to validate how well we are accurately diagnosing carotid stenosis. We also incorporate weighted kappa in our statistical analysis to stress the importance of choosing sensitivity and specificity values to reflect clinical importance, as mentioned in our discussion about weighted kappa.

In choosing US parameter values with the best predictive value, it may help to assess the available data at each institution. 
For example, as a screening test for stenosis, parameter values of PSV, EDV and VR can be chosen such that the test has a higher sensitivity over specificity in order to minimize false negative results. Likewise, it is important to recognize the need for patient safety and financial constraints. For example, a very high sensitivity testing will also create false positive results which may expose the patient to unnecessary further studies like angiogram (DSA) with its inherent risks. False positive results also lead to costly confirmatory studies with additional monetary costs. A recent article by Buskens et $\mathrm{al}^{23}$ suggests that Duplex US without additional imaging is cost effective in selecting symptomatic patients suitable for endarterectomy. For the purpose of our study, we chose to pick the parameter value yielding the best combination of sensitivity and specificity based on ROC curves. Other institutions may choose different values for different reasons.

Other studies have reported that the Doppler ultrasound PSV values for stenosis $>70 \%$, are much less than the current value of $230 \mathrm{~cm} / \mathrm{s}$ used at our institution. Koga et al ${ }^{24}$ reported that PSV of $>200 \mathrm{~cm} / \mathrm{s}$ was the most reliable predictor of ICA stenosis greater than $70 \%$ at their institution. Carpenter et al ${ }^{10}$ describe methods for determining Doppler ultrasound parameters for degree of stenosis $>70 \%$ and also mention the need for vascular laboratories to validate their own results. Their suggested values for stenosis $>70 \%$ are PSV of $>210 \mathrm{~cm} / \mathrm{s}$, EDV $>70 \mathrm{~cm} / \mathrm{s}, \mathrm{VR}>3.0$, and ICA:CCA EDV Ratio $>3.3$. Sabeti et $\mathrm{al}^{25}$ compared 13 sets of published US criteria to look at US parameter agreement with their own institution. In looking at this review of similar studies, our PSV value of $173 \mathrm{~cm} / \mathrm{s}$ for stenosis $>70 \%$ is still somewhat low in comparison to other studies. We are unsure as to why our results for PSV are lower than those reported by these studies. However, this difference may be due to the methods we used to choose our cutoff values to reflect the best combination of sensitivity and specificity as seen at the ROC curve vertex point. Inherent limitations of our study may also have an effect on our parameter values.

While our US parameter value PSV $(173 \mathrm{~cm} / \mathrm{s})$ for stenosis > $70 \%$ yields a higher value for agreement (Table 6), it is interesting to note that our EDV and VR values yield a lower value for agreement. From this we suggest that PSV parameters may be lowered at our institution from $230 \mathrm{~cm} / \mathrm{s}$ to a lower value approaching our value of $173 \mathrm{~cm} / \mathrm{s}$. EDV and VR values need not be changed as they are optimal at our institution. From this it is estimated that a higher sensitivity and specificity can be obtained when correlating US to DSA.

We believe that our study, although similar to others, is unique in the use of weighted kappa statistical evaluation in assigning value and significance to the direction of misclassifications by US when compared to DSA. We have not found other studies which assign weighted kappa values to misclassifications. We believe that future studies should consider the significance of false positive (FP) and false negative (FN) values in terms of which one would be more acceptable (FP or $\mathrm{FN})$ to patients, physicians and medical administrators.

We recognize several limitations of our study which may have some effect on the validity of our results and conclusions. The retrospective nature of the study is an inherent limitation which cannot be changed. The decision for undergoing cerebral angiogram in every patient was made only based on the referring physician preference after reviewing the screening result with the US. This obviously could potentially lead to selection bias (of the patients with more stenosis who are sent for DSA, potentially missing those with true significant stenosis which is shown to have no major stenosis on the US). Another source of bias is the misclassification of patients due to the prior knowledge of the patient history which could unconsciously encourage the ultrasonographer or radiologist to overestimate the degree of stenosis. Another limitation is with the US technique. US is operator dependent, and being a retrospective study, we were unable to correct for the differences between US operators. The inherent limitation of the US correlating percent stenosis to the velocity of blood flow as measured by the Doppler effect, has been mentioned earlier. This limitation will always make US an inferior technique in precisely measuring stenosis, but its value as a screening tool will remain.

The MRA techniques and technology are improving with time, and another limitation of our study is that the MRAs had slight variations between each patient, such as the number of images taken and the size of the images printed to film. For example, it was easier to objectively measure \% stenosis from a large image of the carotid at the point of maximal stenosis than when the images were smaller. Smaller images required more subjective clinical judgement to determine \% stenosis. Further refinement of MRA technique undoubtedly will allow for more accurate evaluation of carotid stenosis.

Despite our limited MRA data, we feel that it is still important mainly to highlight the concept of sequential testing, as well as to stress the validity of MRA. With the new era of CT angiogram becoming the test of choice, the concept of sequential testing can be reapplied and tested in future studies.

CT Angiography (CTA) is an emerging method of screening and diagnosing vascular disorders, including carotid stenosis. ${ }^{26,27}$ A systematic review of CTA has also been done, showing that CTA is accurate for detecting severe carotid artery stenosis, especially for detecting occlusions. ${ }^{9}$ Budget limitations, availability of multidetector CT scanners, and availability of trained technologists continue to be among the constraints to widespread use of CTA. For now, there are limitations of it's use in our centre, and carotid US remains as a predominant screening test for stenosis.

Doppler Ultrasound parameter values used for prediction of carotid stenosis should be validated in each centre using a simple retrospective data analysis such as the one demonstrated in this paper. At our institution, $\mathrm{PSV}$ of $>173 \mathrm{~cm} / \mathrm{s}$ is the best predictor of carotid stenosis $>70 \%$, and sensitivity and specificity of US correlation to DSA can be improved by lowering the current PSV cutoff value from $230 \mathrm{~cm} / \mathrm{s}$ to a value closer to $173 \mathrm{~cm} / \mathrm{s}$.

The MRA had a perfect specificity and PPV in our study. However, in gathering data it became evident that MRA techniques and interpretations needed to be standardized and improved. Further studies are needed to validate MRA in comparison to DSA.

Using US and MRA as simultaneous screening tests, we can increase the overall sensitivity for diagnosing $>70 \%$ stenosis without compromising overall specificity as long as the MRA specificity remains close to $100 \%$.

Kappa values for PSV are slightly higher for higher grade 
stenosis than moderate stenosis, as seen when comparing $>70 \%$ stenosis to $>40 \%$ (0.641 to 0.595 ) (Table 5, 6). Using Kappa alone can be deceiving in assessing the degree of agreement between a screening test such as US and a gold standard diagnostic test such as DSA. In these situations, Asymmetrically Weighted Kappa should be used to validate a screening test result, as it allows us to assign importance to the direction of disagreement.

\section{REFERENCES}

1. Culebras A, Kase CS, Masdeu JC, et al. AHA Scientific Statement. Practice guidelines for the use of imaging in transient ischemic attacks and acute stroke. A report of the stroke council, American Heart Association. Stroke 1997; 28:1480-1497.

2. Dion JE, Gates PC, Fox AJ, et al. Clinical events following neuroangiography: A prospective study. Stroke 1987; 18:9971004.

3. Executive committee for ACAS study. Endarterectomy for asymptomatic carotid artery stenosis. JAMA 1995; 273:14211428.

4. Johnston DCC, Chapman KM, Goldstein LB. Low rate of complications of cerebral angiography in routine clinical practice. Neurology 2001; 57:2012-2014.

5. Leclerc X, Pruvo JP: Recent advances in magnetic resonance angiography of carotid and vertebral arteries. Curr Opin Neurol 2000; 13:75-82.

6. Riles TS, Eidelman EM, Litt AW, et al. Comparison of magnetic resonance angiography, conventional angiography, and duplex scanning. Stroke1992; 23:341-346.

7. Jackson MR, Chang AS, Robles HA, et al. Determination of $60 \%$ or greater carotid stenosis: A prospective comparison of magnetic resonance angiography and duplex ultrasound with conventional angiography. Ann Vasc Surg 1998; 12:236-243.

8. Johnston DCC, Eastwood JD, Nguyen T, Goldstein LB. Contrastenhanced magnetic resonance angiography of carotid arteries. Utility in routine clinical practice. Stroke 2002; 33:2834-2838.

9. Koelemay MJ, Nederkoorn PJ, Reitsma JB, et al. Systematic review of computed tomographic angiography for assessment of carotid artery disease. Stroke 2004; 35:2306-2312.

10. Carpenter JP, Lexa FJ, Davis JT. Determination of duplex doppler ultrasound criteria appropriate to the North American symptomatic carotid endarterectomy trial. Stroke 1996; 27:695-699.

11. Cutnell JD, Johnson KW. Physics, 4th ed. John Wiley \& Sons, Inc. 11:324-329, 1998.

12. Alexandrov AV, Vital D, Brodie DS, et al. Grading carotid stenosis with ultrasound: An interlaboratory comparison. Stroke 1997; 28:1208-1210.

13. Zwiebel WJ. New doppler parameters for carotid stenosis. Seminars in ultrasound, CT and MRI 1997; 18:66-71.

14. NASCET- North American symptomatic carotid endarterectomy trial. Methods, patient characteristics, and progress. Stroke 1991; 22:711-720.
15. NASCET- North American symptomatic carotid endarterectomy trial collaborators. Beneficial effect of carotid endarterectomy in symptomatic patients with high-grade stenosis. N Engl J Med 1991; 325:445-453.

16. Moneta GL, Edwards J M, Chitwood RW, et al. Correlation of North American symptomatic carotid endarterectomy trial (NASCET) Angiographic definition of $70 \%$ to $99 \%$ internal carotid artery stenosis with duplex scanning. J Vasc Surg 1993; 17:152-159.

17. Fox AJ. How to measure carotid stenosis. Radiology 1993; 186:316-318

18. Rothwell PM, Gibson R, Warlow CP, et al. Interrelation between plaque surface morphology and degree of stenosis on carotid angiograms and the risk of ischemic stroke in patients with symptomatic carotid stenosis. Stroke 2000; 31:615-621.

19. Streifler JY, Eliasziw M, Fox AJ, et al. Angiographic detection of carotid plaque ulceration. Comparison with surgical observations in a multicenter study. Stroke 1994; 25:1130-1132.

20. ACAS- The Asymptomatic Carotid Atherosclerosis Study Group. Study design for randomized prospective trial of carotid endarterectomy for asymptomatic atherosclerosis. Stroke 1989; 20:844-849.

21. Portney LG, Watkins MP. Foundations of clinical research application to practice, 2nd ed. Prentice-Hall Inc. 2000;26:568575.

22. Cohen J: Weighted Kappa. Nominal scale agreement with provision for scaled disagreement or partial credit. Psycholo Bull 1968; 70:213-220.

23. Buskens E, Nederkoorn PJ, Buijs-van der Woude T, et al. Imaging of carotid arteries in symptomatic patients: Cost-effectiveness of diagnostic strategies. Radiology 2004; 233:101-112.

24. Koga M, Kimura K, Minematsu K, et al. Diagnosis of internal carotid artery stenosis greater than $70 \%$ with power doppler duplex sonography. AJNR Am J Neuroradiology 2001; 22:413417.

25. Sabeti S, Schillinger M, Wolfgang M, et al. Quantification of internal carotid artery stenosis with duplex US: Comparative analysis of different flow velocity criteria. Radiology 2004; 232:431-439.

26. Herzig R, Burval S, Krupka B, et al. Comparison of ultrasonography, CT angiography, and digital subtraction angiography in severe carotid stenoses. Eur J of Neurol 2004; 11:774-781.

27. Randoux B, Marro B, Marsault C. Carotid artery stenosis: Competition between CT angiography and MR angiography. AJNR Am J Neuroradiol 2004; 25:663-664. 